\title{
Extensive Keloids Following Hair Transplantation
}

\author{
MARC D. BROWN, M.D. \\ TIMOTHY JOHNSON, M.D. \\ NEIL A. SWANSON, M.D.
}

\begin{abstract}
Hair transplant surgery is a highly successful procedure for improving alopecia. However, this procedure is not without its potential risks and complications. A case of a large keloidal scar following hair transplantation is presented. Those performing hair transplant surgery should be aware of this significant potential complication, especially in Black and Oriental individuals, who are more prone to keloid formation. I Dermatol Surg Oncol 1990; 16:867-869
\end{abstract}

\section{INTRODUCTION}

Hair transplant surgery is a highly successful procedure for improving alopecia, especially malepattern alopecia. This surgical technique is available to all dermatologists who have a cosmetic surgical interest, and the technique continues to be refined and improved by those who perform it on a regular basis. However, this procedure is not without its potential risks and complications. Described adverse effects include the following: bleeding, infection, edema, A.V. malformations, pyogenic granuloma, cobble-

Marc D. Brown, M.D., is Assistant Professor of Dermatology, Department of Dermatology, University of Rochester, Rochester, New York.

Timothy Johnson, M.D., Assistant Professor and Neil A. Swanson, M.D., Associate Professor of Dermatology and Otorhinolaryngology, are from the Department of Dermatology, the University of Michigan Medical Center, Ann Arbor, Michigan.

Address reprint requests to Marc D. Brown, M.D., Department of Dermatology, University of Rochester, 601 Elmwood Ave., Rochester, NY 14642.

(C) 1990 by Elsevier Science Publishing Co., Inc. $\bullet 0148-0812 / 90 / \$ 3.50$ stoning of the plugs at the recipient site, osteomyelitis, and poor hair growth from the graft. ${ }^{1}$ We encountered a rather dramatic untoward effect of hair transplant surgery: a large keloidal scar following hair transplantation. We present this case for the express purpose of reminding those who perform this procedure to be aware of this significant potential complication, especially in Black and Oriental individuals, who are more prone to keloid formation.

\section{CASE REPORT}

A Black male developed male pattern alopecia in his early twenties. At the age of 60 years, he sought correction of his alopecia with hair transplant surgery. The initial surgical procedure was performed by another physician with the left and right occipital scalp serving as the donor sites. The procedure was initially without complication and immediate postoperative healing was good, according to the patient's history. There was no untoward bleeding or secondary infection. The donor site was allowed to heal by second intention. Several months later, the patient began to develop a large keloidal scar at the occipital donor site. In addition, smaller keloids were noticed at several of the recipient sites in the frontal hairline. Of note, the patient gave no previous history of keloidal scar formation, and there were no unusual predisposing factors other than race. The patient was in good general health.

Treatment with high-dose intralesional triamcinolone acetate injections $(40 \mathrm{mg} / \mathrm{cc}$ ) as well as cryotherapy were not helpful. We first saw the patient for evaluation and possible treatment of his occipital keloid with $\mathrm{CO}_{2}$ laser surgery. Figures 1 and 2 show the 


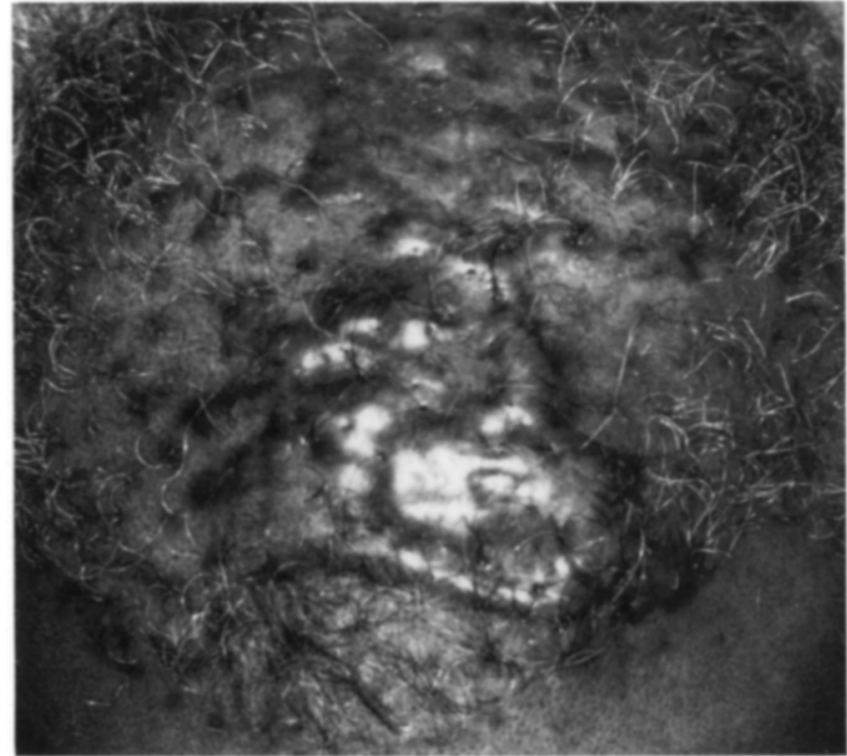

FIGURE 1. Keloidal scar at recipient site.

patient prior to his laser surgery. As one can see, he had an extensive keloidal scar extending across the entire occipital area, as well as smaller keloidal scars on the top of his scalp at the recipient sites. The patient was subsequently treated with $\mathrm{CO}_{2}$ laser excision of these keloids. To date, he has had four laser surgical procedures performed on this large occipital keloid (Figs. 3 and 4). Intralesional triamcinolone acetate $(10 \mathrm{mg} / \mathrm{cc})$ was used in conjunction with each surgical laser procedure in order to decrease the chance of keloid recurrence.

The results have been quite encouraging. There has been some postsurgical hypopigmentation and

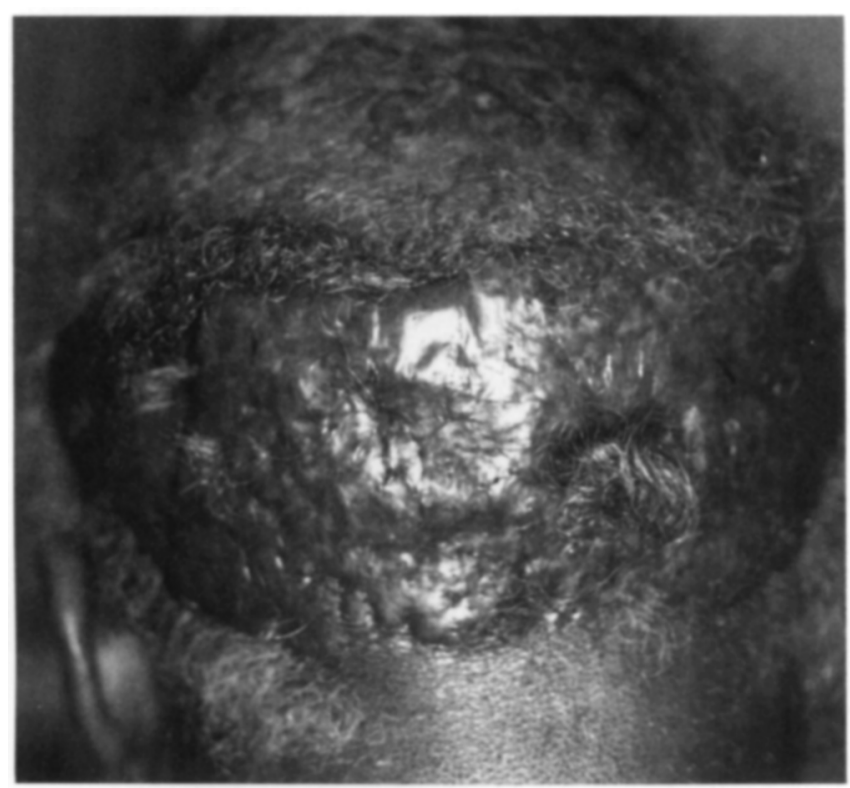

FIGURE 2. Keloidal scar at donor site.

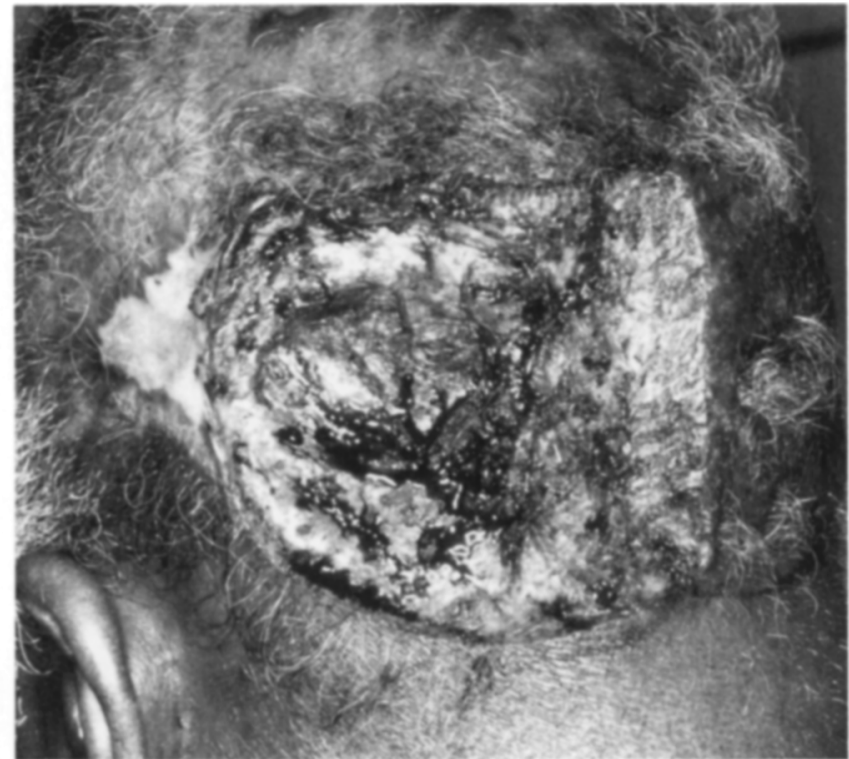

FIGURE 3. First-stage laser excision of occipital scalp keloid.

some small recurrent keloids, which responded nicely to intralesional triamcinolone injections. Figure 5 shows the most recent status of the patient with a generally acceptable result, although the patient realizes that there will never be any hair growth in this area and that hypopigmentary changes may be permanent.

\section{DISCUSSION}

Keloidal scarring is a poorly understood healing phenomenon. Pathogenesis is probably related to alter-

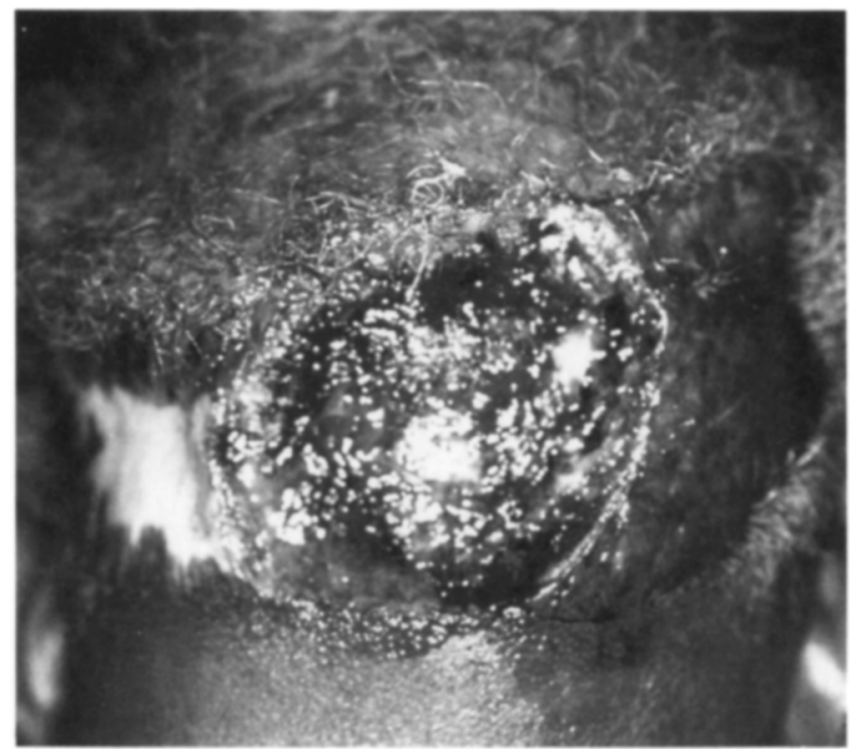

FIGURE 4. Second-stage laser excision of occipital scalp keloid. 


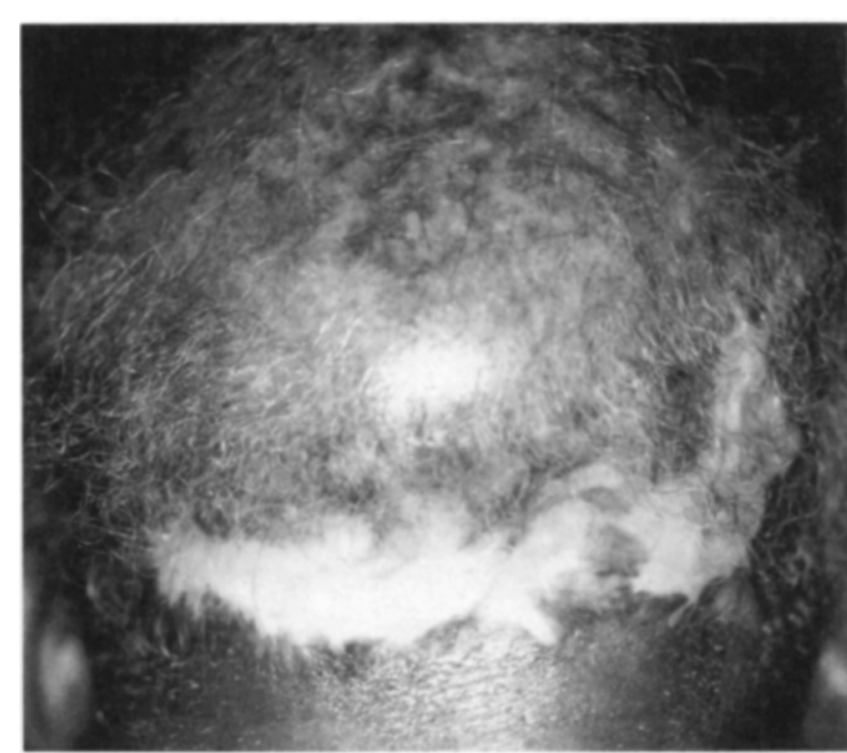

FIGURE 5. Hypopigmented flat scar after four laser excisional procedures.

ations in collagen synthesis and deviation of normal fibroblastic growth. ${ }^{2}$ Keloids usually follow a traumatic and/or inflammatory event and are more frequent in dark-skinned individuals, with approximately $75 \%$ of keloids occurring in Blacks. It is more commonly encountered in certain anatomic locations, such as the chest, upper back, earlobes, neck, and shoulders. By definition, the keloidal scar grows over the boundary of the initial scar or site of trauma. Keloids can be both painful and pruritic, as was the case in our patient. The growth and final size of a keloid is difficult to predict. It is also difficult to predict which individual patient will develop a keloid.

Our patient sought medical attention for the surgical correction of male pattern alopecia. Unfortunately, the end result was a cosmetically unacceptable keloidal scar involving a large portion of his scalp. In his textbook on hair transplantation, Unger points out that because Black patients are known to be more prone to keloid formation, he always conducts a test graft session prior to a full transplant procedure. ${ }^{1} \mathrm{~A}$ single, 4.0-mm graft is removed from the donor area and placed adjacent to the fringe area of the potential recipient zone, where it will be least noticeable if keloidal formation occurs. There is then a waiting period of 3 months to see if healing is normal before proceeding with full-punch transplanting sessions. Certainly, if there is a history of keloid formation, hair transplant surgery is contraindicated. However, it does remain a possibility that even if the test site heals well, the patient may still develop a keloid when the full procedure is performed. For this reason, some hair transplant surgeons do not advise a test graft session because it does not reliably predict those patients who will develop keloids.

There exists a widespread belief that Black patients are not good cosmetic surgery candidates because of the perceived inevitability of scarring problems. Actually, most Black patients have excellent cosmetic results from hair transplant surgery and do not exhibit keloidal scarring. We present this case to stress two important points. First, as cosmetic surgeons, we need to be acutely aware of unusual as well as usual adverse effects due to any surgical procedure. Second, it is imperative to spend time with the patient preoperatively to carefully explain all of the possible complications of a given cosmetic surgical procedure. Certainly, with careful patient selection and attention to surgical detail, results are usually excellent. However, disastrous results unrelated to our surgical skill or postoperative care may occur. Extensive keloidal scarring secondary to hair transplantation is such an example that we need to be mindful of and warn patients about.

\section{REFERENCES}

1. Unger W, Nordstrom R. Hair Transplantation. New York, Marcel Dekker, 1988.

2. Bennett R. Fundamentals of Cutaneous Surgery. St. Louis, CV Mosby, 1988, pp 709-719. 